\title{
Analysis of preoperative circulating tumor cells for recurrence in patients with hepatocellular carcinoma after liver transplantation
}

\author{
Zhitao Chen ${ }^{1,2,3 \#}$, Xiaohong Lin ${ }^{1,2,3,4 \#}$, Chuanbao Chen ${ }^{1,2,3 \#}$, Yinghua Chen ${ }^{1,2,3}$, Qiang Zhao ${ }^{1,2,3}$, \\ Linwei Wu ${ }^{1,2,3}$, Dongping Wang ${ }^{1,2,3}$, Yi Ma ${ }^{1,2,3}$, Weiqiang Ju ${ }^{1,2,3}$, Maogen Chen $^{1,2,3}$, Xiaoshun $\mathrm{He}^{1,2,3}$ \\ ${ }^{1}$ Organ Transplant Center, First Affiliated Hospital of Sun Yat-sen University, Guangzhou, China; ${ }^{2}$ Guangdong Provincial Key Laboratory of Organ \\ Donation and Transplant Immunology, Guangzhou, China; ${ }^{3}$ Guangdong Provincial International Cooperation Base of Science and Technology \\ (Organ Transplantation), Guangzhou, China; ${ }^{4}$ Division of General Surgery, The Eastern Hospital of the First Affiliated Hospital of Sun Yat-sen \\ University, Guangzhou, China \\ Contributions: (I) Conception and design: X He, M Chen; (II) Administrative support: M Chen, W Ju; (III) Provision of study materials or patients: \\ Y Chen, Q Zhao, L Wu, D Wang, Y Ma; (IV) Collection and assembly of data: Z Chen, C Chen; (V) Data analysis and interpretation: X Lin; (VI) \\ Manuscript writing: All authors; (VII) Final approval of manuscript: All authors. \\ \#These authors contributed equally to this work. \\ Correspondence to: Maogen Chen; Weiqiang Ju; Xiaoshun He. Division of Organ Transplant Center, First Affiliated Hospital of Sun Yat-sen \\ University, Guangzhou, China. Email: chenmg3@mail.sysu.edu.cn; weiqiangju@163.com; gdtrc@163.com.
}

Background: Liver transplantation (LT) is an effective option for hepatocellular carcinoma (HCC) and end-stage liver cirrhosis. However, organ shortage and tumor recurrence are the main obstacles limiting its popularization and application in HCC patients. Testing for circulating tumor cells (CTCs) would be a valuable strategy to predict the recurrence and metastasis of HCC after LT. Various CTCs detection methods have different advantages and disadvantages. This study aims to investigate the predictive value of testing for CTCs based on immunofluorescence in situ hybridization of peripheral blood cells in patients with HCC after LT.

Methods: A total of 50 patients who received testing for CTCs and then underwent LT were enrolled in this study. Negative enrichment and immunofluorescence in situ hybridization (imFISH) methods were introduced to detect CTCs.

Results: Twenty-six (52\%) patients were CTC-positive, and 24 (48\%) patients were CTC-negative. The results showed that CTCs result was correlated with tumor size $\left(\chi^{2}=5.773, \mathrm{P}=0.016\right)$, AFP level $\left(\chi^{2}=5.454, \mathrm{P}=0.020\right)$, tumor grade $\left(\chi^{2}=6.478, \mathrm{P}=0.039\right)$ and $\operatorname{Recurrence}\left(\chi^{2}=6.211, \mathrm{P}=0.013\right)$. Twelve patients had recurrence after LT within one year. The results showed that the CTCs result $(\mathrm{P}=0.034)$ was the only independent factor impacting long-term survival. The 1-year disease-free survival rates of CTC-negative and CTC-positive patients were $91.6 \%$ and $61.5 \%$, respectively $(\mathrm{P}=0.020)$. The 1 -year overall survival of CTCpositive patients and CTC-negative was $88.5 \%$ and $91.7 \%$, respectively $(\mathrm{P}=0.751)$.

Conclusions: CTCs result was closely related to the early recurrence of patients with HCC after LT. CTC-positive patients had a worse prognosis after LT than the CTC-negative group.

Keywords: Circulating tumor cell (CTC); hepatocellular carcinoma (HCC); liver transplantation (LT)

Submitted Mar 22, 2020. Accepted for publication Jul 27, 2020.

doi: $10.21037 /$ atm-20-2751

View this article at: http://dx.doi.org/10.21037/atm-20-2751 


\section{Introduction}

Hepatocellular carcinoma (HCC) is a malignant disease with high morbidity and mortality. In the world, HCC is the fifth most common and third most deadly cancer, and it is the third leading cause of cancer-related death in China (1). Hepatectomy was once the standard treatment to cure HCC worldwide. However, according to some studies, even with radical resection, the overall 5 -year recurrence rates of large hepatocellular carcinoma (LHC) and small hepatocellular carcinoma (SHC) are $80 \%$ and $43.5 \%$, respectively (2). The main cause of recurrence is tumor cell dissemination via blood vessel infiltration. Circulating tumor cells (CTCs) are likely to spread before or after operation without a definite detection method. In addition, squeezing and destroying tumors during operation is also an important factor leading to hematogenous dissemination of tumor cells.

CTCs were first discovered in the blood of a patient with metastatic cancer and described by Ashworth et al. in 1869 (3). With nearly 100 years of improvements, the predictive value of CTCs has been emphasized. In 1998, Racila et al. introduced a new method to detect CTCs and suggested that CTCs appeared early and were associated with tumor progression, creating the theoretical basis for the clinical use of CTCs in current cancer research and therapy (4). Vona et al. first investigated the prognostic value of blood CTCs in patients with HCC and showed a close association with prognosis and recurrence in patients with HCC (5). Multifarious approaches were developed for detection, and various CTC methods result in different results. At present, the existing CTC detection methods are as follows: Immunoaffinity-based positive enrichment (6) (antibodies target tumor cell-associated antigens and then adhere to magnetic particles), immunoaffinity-based negative enrichment (7) (antibodies target unwanted background cells and then adhere to magnetic particles), biophysical separation methods [cells are separated by biophysical characteristics such as density (8)], direct imaging modalities (a series of novel methods developed to replace enrichment), functional assays (enrichment is based on cell bioactivity), and so on.

Liver transplantation (LT) is an effective treatment option for both tumors and cirrhosis (9). Patients who meet the Milan criteria and the University of California San Francisco (UCSF) criteria can achieve encouraging survival outcomes after LT. Saposochin et al. and Bruix et al. reported that the 5-year post-transplantation overall survival rate could reach $75 \%$ for patients with HCC meeting the criteria (10). However, organ shortage and recurrence limited the selection of LT treatment in patients with HCC. Prognoses of tumor recurrence based on CTCs are meaningful for the selection of LT patients and followup observations. This retrospective study aims to investigate the predictive value of testing for CTCs for early recurrence of patients with HCC after LT. We present the following article in accordance with the STROBE reporting checklist (available at http://dx.doi.org/10.21037/atm-20-2751).

\section{Methods}

The study was conducted in accordance with the Declaration of Helsinki (as revised in 2013) and approved by the Institutional Ethics Committee for Clinical Research and Animal Trials of the First Affiliated Hospital of Sun Yatsen University and informed consent waiver was granted by the IEC given the retrospective, minimal risk nature of the study \{Approval ID: [2020]088\}.

\section{General information}

Three hundred and twenty-eight patients were diagnosed and then underwent LT in our department from January $1^{\text {st }}, 2016$ to February $28^{\text {th }}, 2019$. Among them, a total of 94 patients who were tested for CTCs were enrolled. The inclusion criteria were as follows: 18 to 75 years of age, a diagnosis of HCC confirmed by postoperative pathological examination, and follow-up of more than 1 year. The exclusion criteria were as follows: patients with perioperative or nonrecurrence-related mortality, a diagnosis of other types of tumors, and follow-up of less than 1 year. Three patients' postoperative pathological diagnoses were without tumors, 9 patients died within 30 days of operation and 32 patients' follow-up periods were less than 1 years. Afterwards, 50 patients were included in this study. The immunosuppressive regimen was tacrolimus (Tac) + mycophenolate mofetil (MMF). Corticosteroid was not included in routine regimen for its side effects.

\section{CTCs detection}

The samples for CTCs analysis were collected at preLT. Negative enrichment and imFISH methods were introduced to detect CTCs. Peripheral blood $(3.2 \mathrm{~mL})$ was processed by a Human Blood Cell Deletion Kit (Cyttel). First, red blood cells were lysed and depleted. The residual cell pellet was suspended again and incubated with anti- 


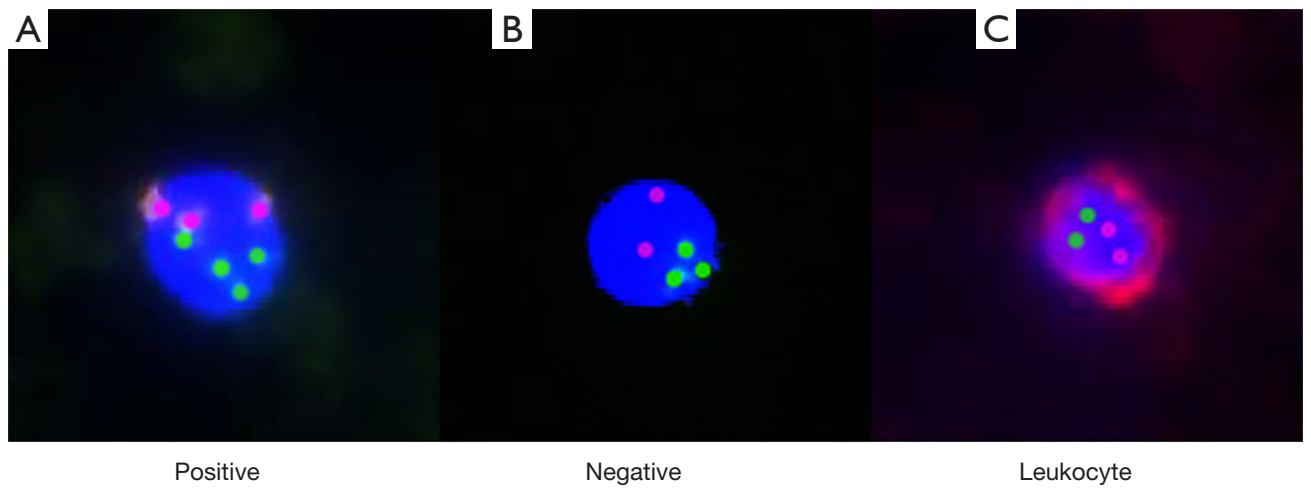

Figure 1 Preoperative CTC fluorescence imaging in patients with HCC. The phenotype of CTCs was CEP8+/DAPI+/CD45- (DAPI: blue; CEP8: orange; CD45: red). When the CEP8 signal was $\geq 3$, it was considered positive (A). When the CEP8 signal was $\leq 2$, it was considered negative (B). The phenotype of leukocytes was CEP8+/CD45+/DAPI+ (C).

CD45 monoclonal antibody-coated magnetic beads for 20 30 minutes. Then, A magnetic stand (Promega, Madison, WI) was used to separate these beads. The supernatant was subsequently used for identification.

The identification of enriched CTCs was performed by imFISH, which combined the FISH probes with chromosome 8 (orange) centromere probes (Abbott Molecular Diagnostics, Des Plaines, IL, USA) and antiCD45 monoclonal antibodies (Red, Cyttel). First, the CEP8 probe and specimen were hybridized at $37{ }^{\circ} \mathrm{C}$ for 20-90 minutes in a hybridizer (DAKO). Subsequently, they were washed in $50 \%$ formamide at $43{ }^{\circ} \mathrm{C}$ for 15 minutes and then subjected to gradient alcohol. Finally, the specimens were incubated with anti-CD45 antibodies conjugated to Alexa Flour 594 (Invitrogen) for 1 hour. Afterwards, they were washed again with $0.2 \%$ BSA. Finally, the specimens were stained with DAPI, which contained Vectashield mounting medium. To be considered positive, CTCs needed to be hyperdiploid and have the phenotype CEP8+/ DAPI+/CD45-. The CTC test results are shown in Figure 1.

\section{Follow-up}

The patients were followed up for at least 1 year. For deceased patients/patients with recurrences, the date of death/recurrence was used as the last follow-up. Postoperative visits were performed on postoperative day (POD) 1-7, POD14 and each postoperative month (POM). Biomedical values, imaging examinations and tumor markers were documented. Routine Doppler ultrasound of the liver graft blood flow and biliary tract was performed once every 2 days for 7 days. Afterward, imaging studies were performed based on patients' clinical status or laboratory findings. The follow-up deadline was February $29^{\text {th }}, 2020$.

\section{Statistical analysis}

All statistical analyses of the data were performed by SPSS version 26.0. All data are expressed as the mean \pm standard deviation or the number and percentage of patients. For comparison between groups, the chi-square and Fisher's exact tests were performed for frequencies and continuous data, respectively. Cox proportional hazards model was performed for multivariate analysis. Overall and diseasefree survival were compared using the Kaplan-Meier method with a log-rank test. A $P$ value $<0.05$ was considered statistically significant.

\section{Result}

\section{Patient characteristics}

To eliminate selection bias, we compared baseline data between CTC-test group and no CTC-test group (Table 1) and there were no significant differences between groups $(\mathrm{P}>0.05)$. A total of 50 patients who were tested for CTCs and then underwent LT were analyzed, and their characteristics are shown in Tables 2 and 3. The male-to-female ratio was 24 to 1 . The mean age was $52.88 \pm 1.43$ years old. Forty-seven $(94 \%)$ patients had cirrhosis, and 38 (76\%) patients had hepatitis B virus (HBV) infection. Nineteen (38\%) patients had larger tumors $(>6.5 \mathrm{~cm})$ and $5(10 \%), 24(48 \%)$, and $21(42 \%)$ patients 
Table 1 Comparison of baseline data between CTC-test group and no CTC-test group

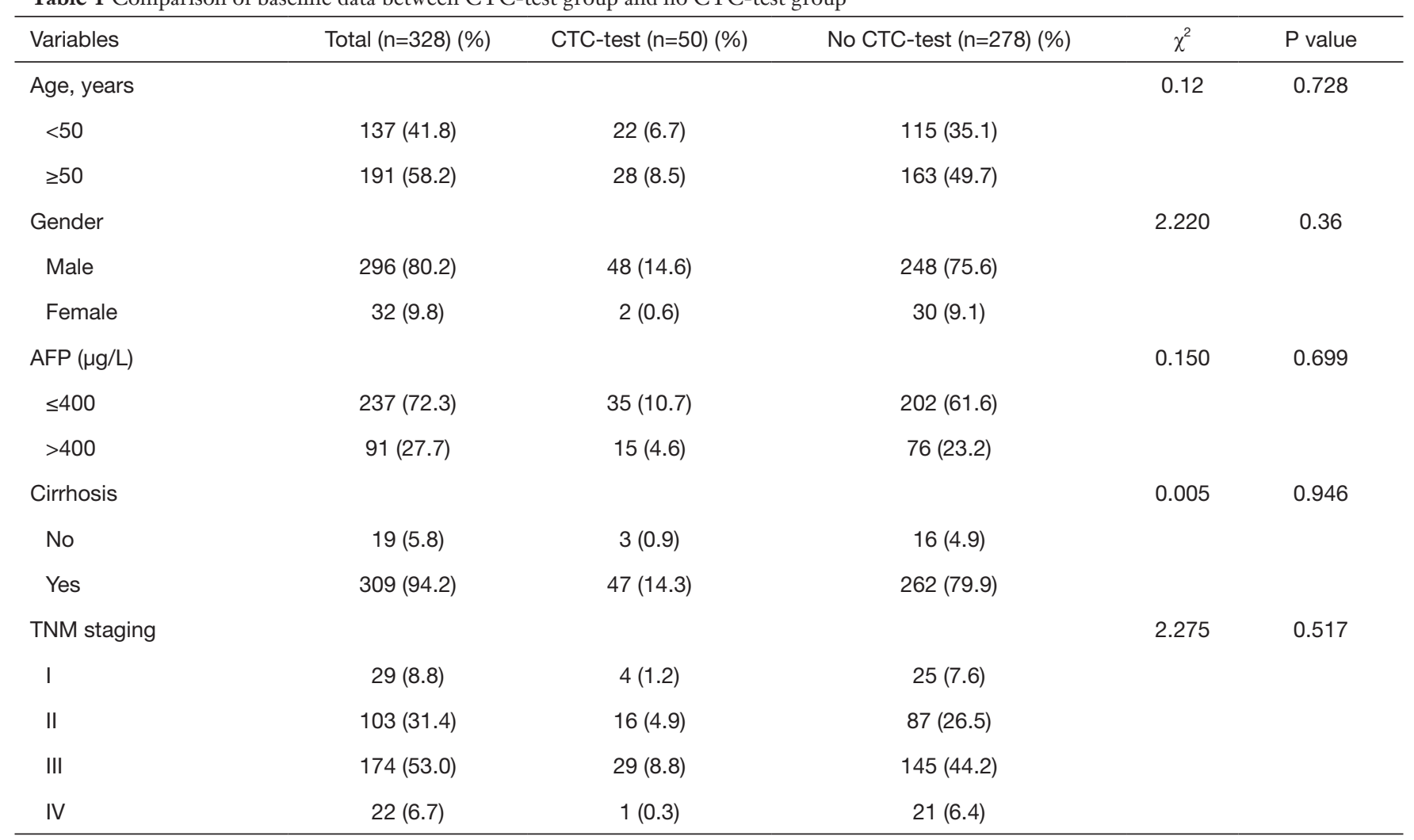

CTC, circulating tumor cells; AFP, preoperative alpha-fetoprotein.

Table 2 Association between peripheral blood CTCs count and baseline data of 50 patients

\begin{tabular}{|c|c|c|c|c|c|}
\hline \multirow{2}{*}{ Variables } & \multirow{2}{*}{ Count (\%) } & \multicolumn{2}{|c|}{ Peripheral blood CTCs count (\%) } & \multirow{2}{*}{$\chi^{2}$} & \multirow{2}{*}{$P$ value } \\
\hline & & $>1$ & 0 & & \\
\hline Age, years & & & & 0.102 & 0.746 \\
\hline$\geq 50$ & $28(8.5)$ & $14(4.3)$ & $14(4.3)$ & & \\
\hline \multicolumn{6}{|l|}{ Gender } \\
\hline Female & $2(0.6)$ & $1(0.3)$ & $1(0.3)$ & & \\
\hline Tumor size $(\mathrm{cm})$ & & & & 5.773 & 0.016 \\
\hline$\leq 6.5$ & $31(9.5)$ & $12(3.7)$ & $19(5.8)$ & & \\
\hline$>6.5$ & $19(5.8)$ & $14(4.3)$ & $5(1.5)$ & & \\
\hline PVT & & & & 0.056 & 0.814 \\
\hline No & $41(12.5)$ & $21(6.4)$ & $20(6.1)$ & & \\
\hline Yes & $9(2.7)$ & $5(1.5)$ & $4(1.2)$ & & \\
\hline
\end{tabular}

Table 2 (continued) 
Table 2 (continued)

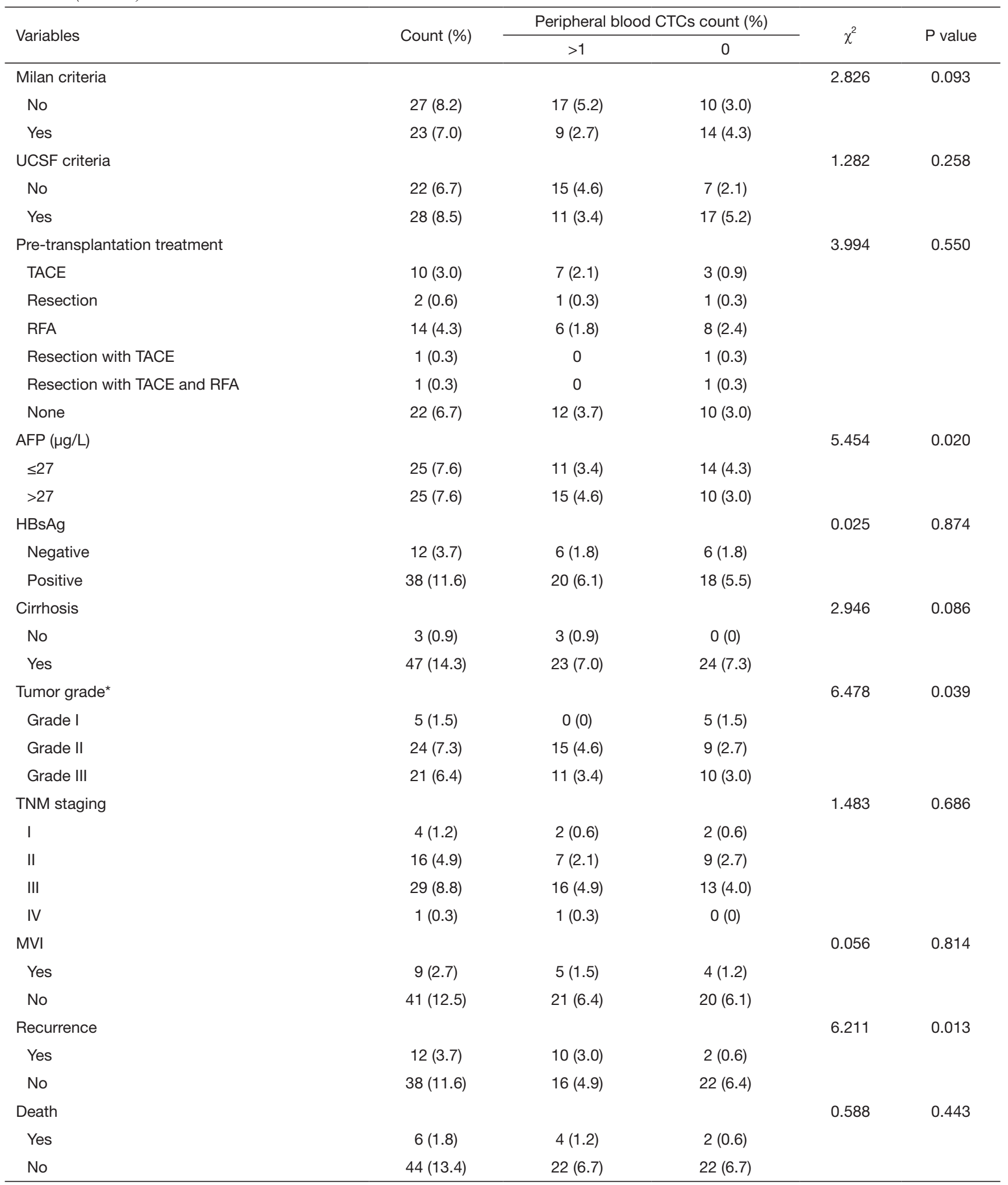

*, HCC cellular differentiation. CTC, circulating tumor cells; PVT, portal vein thrombosis; TACE, transarterial chemoembolization; RFA, radiofrequency ablation; AFP, preoperative alpha-fetoproteins; HBsAg, hepatitis B surface antigen; MVI, microvascular invasions. 
Table 3 Analysis of relevant factors for recurrence of HCC in 50 patients

\begin{tabular}{|c|c|c|c|c|c|}
\hline Variables & Count (\%) & \multicolumn{2}{|c|}{ Recurrence (\%) } & $\chi^{2}$ & $P$ value \\
\hline Age, years & & & & 1.317 & 0.251 \\
\hline$<50$ & $22(6.7)$ & $7(2.1)$ & $15(4.6)$ & & \\
\hline$\geq 50$ & $28(8.5)$ & $5(1.5)$ & $23(7.0)$ & & \\
\hline Male & $48(14.6)$ & $12(3.7)$ & $36(11.0)$ & & \\
\hline Female & $2(0.6)$ & $0(0)$ & $2(0.6)$ & & \\
\hline Tumor size $(\mathrm{cm})$ & & & & 13.773 & 0.000 \\
\hline$\leq 6.5$ & $31(9.5)$ & $2(0.6)$ & $29(8.8)$ & & \\
\hline$\leq 3$ & 39 (11.9) & $7(2.1)$ & $32(9.8)$ & & \\
\hline$>3$ & $11(3.4)$ & $5(1.5)$ & $6(1.8)$ & & \\
\hline PVT & & & & 2.515 & 0.113 \\
\hline No & $41(12.5)$ & $8(2.4)$ & $33(10.1)$ & & \\
\hline Yes & $9(2.7)$ & $4(1.2)$ & $5(1.5)$ & & \\
\hline Milan criteria & & & & 5.469 & 0.019 \\
\hline No & $27(8.2)$ & $10(3.0)$ & $17(5.2)$ & & \\
\hline Yes & $23(7.0)$ & $2(0.6)$ & $21(6.4)$ & & \\
\hline Resection & $2(0.6)$ & $1(0.3)$ & $1(0.3)$ & & \\
\hline RFA & $14(4.3)$ & $1(20.3)$ & $13(4.0)$ & & \\
\hline Resection with TACE & $1(0.3)$ & 0 & $1(0.3)$ & & \\
\hline Resection with TACE and RFA & $1(0.3)$ & 0 & $1(0.3)$ & & \\
\hline None & $22(6.7)$ & $7(2.1)$ & $15(4.6)$ & & \\
\hline AFP $(\mu g / L)$ & & & & 1.754 & 0.185 \\
\hline$\leq 27$ & $25(7.6)$ & $4(1.2)$ & $21(6.4)$ & & \\
\hline$>27$ & $25(7.6)$ & $8(2.4)$ & $17(5.2)$ & & \\
\hline HBsAg & & & & 0.010 & 0.921 \\
\hline Negative & $12(3.7)$ & $3(0.9)$ & $9(2.7)$ & & \\
\hline Positive & $38(11.6)$ & $9(2.7)$ & $25(7.6)$ & & \\
\hline
\end{tabular}

Table 3 (continued) 
Table 3 (continued)

\begin{tabular}{|c|c|c|c|c|c|}
\hline Variables & Count (\%) & \multicolumn{2}{|c|}{ Recurrence (\%) } & $\chi^{2}$ & $P$ value \\
\hline Cirrhosis & & & & 3.185 & 0.074 \\
\hline No & $3(0.3)$ & $2(0.6)$ & $1(0.3)$ & & \\
\hline Yes & 47 (14.3) & $10(3.0)$ & 37 (11.3) & & \\
\hline Grade I & $5(1.5)$ & $0(0)$ & $5(1.5)$ & & \\
\hline Grade II & $24(7.3)$ & $6(1.8)$ & $18(5.5)$ & & \\
\hline Grade III & $21(6.4)$ & $6(1.8)$ & $15(4.6)$ & & \\
\hline TNM staging & & & & 4.486 & 0.214 \\
\hline III & $29(8.8)$ & $10(3.0)$ & $19(5.8)$ & & \\
\hline IV & $1(0.3)$ & 0 & $1(0.3)$ & & \\
\hline CTCs & & & & 6.211 & 0.013 \\
\hline$>1$ & $26(7.9)$ & $10(3.0)$ & $16(4.9)$ & & \\
\hline 0 & $24(7.3)$ & $2(0.6)$ & $22(6.7)$ & & \\
\hline MVI & & & & 2.515 & 0.113 \\
\hline Yes & $9(2.7)$ & $4(1.2)$ & $5(1.5)$ & & \\
\hline No & 41 (12.5) & $8(2.4)$ & 33 (10.0) & & \\
\hline
\end{tabular}

*, HCC cellular differentiation. HCC, hepatocellular carcinoma; PVT, portal vein thrombosis; TACE, transarterial chemoembolization; RFA, radiofrequency ablation; AFP, preoperative alpha-fetoproteins; HBsAg, hepatitis B surface antigen; CTC, circulating tumor cells.

had tumors classified as grade I, grade II and grade III, respectively (11). The median alpha fetoprotein (AFP) levels in these 50 patients was $27 \mu \mathrm{g} / \mathrm{L}$. Four, 16, 29 and 1 patients had tumors classified as stage I, II, II and IV, respectively. The median follow-up time was 16.4 months (IQR, 15.4-25.55) for recurrence and 20.0 months (IQR, 12.2824.07) for survival, respectively.

\section{CTCs result}

The data of 50 patients were analyzed and are shown in Table 2. Twenty-six (52\%) patients were CTC-positive (CTCs >1), and 24 (48\%) patients were CTC-negative (CTCs $\leq 1)$. The mean ages of the CTC-positive group and CTC-negative group were $52.31 \pm 1.97$ years old and $53.50 \pm 2.12$ years old, respectively. The median preoperative AFP levels of the CTC-positive group and CTC-negative group were 109.0 and $15.9 \mu \mathrm{g} / \mathrm{L}$, respectively. The results showed that CTCs result was correlated with tumor size $\left(\chi^{2}=5.773, \mathrm{P}=0.016\right)$, AFP level $\left(\chi^{2}=5.454, \mathrm{P}=0.020\right)$, tumor grade $\left(\chi^{2}=6.478, \mathrm{P}=0.039\right)$ and Recurrence $\left(\chi^{2}=6.211\right.$, $\mathrm{P}=0.013)$.

\section{Analysis of recurrence factors after $L T$}

Analysis of the 50 patients revealed that 12 (24\%) patients had a recurrence after LT (Table 3). All 12 patients were male, and the mean age was $50.67 \pm 3.39$ years old. The mean preoperative AFP levels of the recurrence group and non-recurrence group were $115,840.99 \pm 111,306.46$ and $945.82 \pm 573.35 \mu \mathrm{g} / \mathrm{L}$, respectively. Ten of these 12 patients had cirrhosis. Ten of these 12 patients were positive for CTCs. The results showed that recurrence was correlated with tumor size $\left(\chi^{2}=13.733, \mathrm{P}<0.001\right)$, compliance with Milan criteria $\left(\chi^{2}=5.469, \mathrm{P}=0.019\right)$, compliance with USCF criteria $\left(\chi^{2}=9.914, \mathrm{P}=0.002\right)$, and the CTCs result $\left(\chi^{2}=6.211\right.$, 
Table 4 Multivariate analysis of relevant factors for recurrence of HCC in 50 patients

\begin{tabular}{lcc}
\hline \multirow{2}{*}{ Variable } & \multicolumn{2}{c}{ Multivariate analysis } \\
\cline { 2 - 3 } & $\mathrm{RR}(95 \% \mathrm{Cl})$ & P value \\
\hline AFP & $2.018(0.591-6.891)$ & 0.263 \\
CTCs & $5.411(1.132-25.874)$ & 0.034 \\
Pre-transplantation & $0.505(0.154-1.660)$ & 0.261 \\
intervention & $1.395(0.450-4.329)$ & 0.564 \\
Tumor grade* & $1.769(0.745-4.200)$ & 0.196 \\
\hline
\end{tabular}

*, HCC cellular differentiation. HCC, hepatocellular carcinoma; AFP, preoperative alpha-fetoproteins; CTCs, circulating tumor cells; MVI, microvascular invasions.

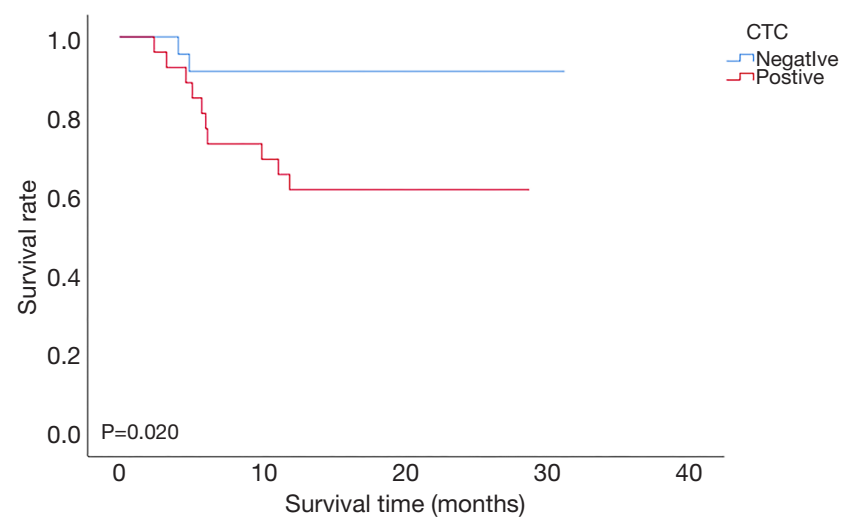

Figure 2 Comparison of disease-free survival between the CTCpositive group and the CTC-negative group in patients with $\mathrm{HCC}$ after LT. CTC, circulating tumor cell; HCC, hepatocellular carcinoma.

$\mathrm{P}=0.013$ ), while there were no significant differences in other groups like preoperative $\mathrm{AFP}\left(\chi^{2}=1.754, \mathrm{P}=0.185\right)$. In multivariate analysis, CTCs result was the only independent impact factor [CTCs: $\mathrm{P}=0.0 .34$; preoperative AFP: $\mathrm{P}=0.263$; Pre-transplantation intervention: $\mathrm{P}=0.261$; Tumor grade: $\mathrm{P}=0.564$; microvascular invasions (MVI): $\mathrm{P}=0.196$ ] (Table 4).

\section{Analysis of disease-free survival and overall survival after $L T$}

During the 12-month follow-up period, two of 24 CTCnegative patients and 10 of 26 CTC-positive patients experienced recurrence. The mean disease-free survival times of CTC-negative and CTC-positive patients were $28.75 \pm 1.56$ and $20.14 \pm 2.13$ months, respectively. The

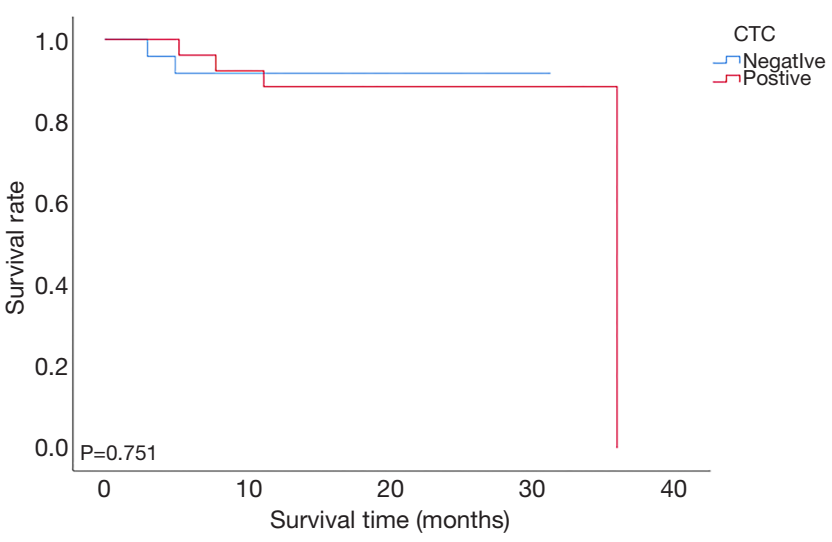

Figure 3 Comparison of overall survival between the CTC-positive group and the CTC-negative group in patients with HCC after LT. CTC, circulating tumor cell; HCC, hepatocellular carcinoma.

1-year disease-free survival rates of CTC-negative and CTC-positive patients were $91.6 \%$ and $61.5 \%$, respectively (91.6\% vs. $61.5 \%, \mathrm{P}=0.020$, Figure 2). The mean overall survival times of CTC-negative and CTC-positive patients were $28.81 \pm 1.53$ and $32.50 \pm 2.0$ months, respectively. The 1 -year overall survival rate of patients with CTC-positive was similar to those with CTC-negative (88.5\% vs. $91.7 \%$, $\mathrm{P}=0.751$, Figure 3). Thirty-two patients whose follow up periods were less than 1 year did not suffer from deaths or recurrences.

\section{Discussion}

Hepatectomy was once the standard treatment to cure HCC worldwide. However, even with complete resection, the recurrence after hepatectomy remains high. LT is an attractive option to cure HCC completely. It can offer R0 resection and achieve the widest negative incisal margin (12). Early studies revealed poor outcomes after LT for HCC related to tumor pathological staging (13). Several advances have been made in the field of LT over decades. With the development of selection criteria such as the Milan criteria and the UCSF criteria (14), technical advances, and enhanced knowledge of HCC staging and biology, the therapeutic efficacy of LT for HCC has achieved an improvement in the 5-year survival rate (15). Approximately $40 \%$ of LT recipients are HCC patients, which means that HCC has been a frequent indication for LT $(16,17)$. However, obstacles exist and affect the prognosis of LT for HCC. Organ shortage has become a worldwide issue, with many patients dying on the waiting list $(18,19)$. For patients 
with HCC, finding a method to predict the prognosis after LT is of great significance. In this study, we aimed to investigate the predictive value of testing for CTCs for the early recurrence of patients with HCC after LT.

First, we analyzed the baseline data of 50 patients. The patients were mostly male and had been diagnosed in their 50s. A large proportion of the tumors developed because of liver cirrhosis with chronic HBV infection and were in an advanced stage when discovered. The reason for these findings may be that in China, patients tend to undergo surgical resection or conservative treatment due to economic or ideological reasons, even if tumors are detected early. LT would be considered only when other treatments were ineffective or if the tumor progressed.

Testing for CTCs is a new noninvasive examination method for early diagnosis, determination of treatment options and prognostic evaluation in HCC due to the continuous improvements in methods related to cell separation and identification (20). Immunoaffinity-based positive enrichment (as also called positive enrichment) and immunoaffinity-based negative enrichment (as also called negative enrichment) are commonly used to detect CTCs. Positive enrichment uses antibodies to target tumor cell-associated antigens, which then adhere to magnetic particles, so tumor cells are captured. Epithelia cell adhesion molecule (EpCAM) is usually used as target antigen to capture CTCs, like the processed used by the Cell Search System (21). This system was approved by the FDA to monitor patients with metastatic solid cancers. However, the lower expression of EpCAM in HCC than in other solid tumors, such as breast cancer and colorectal cancer (22), results in false negative outcomes. Yamashita et al. reported that only $35 \%$ of HCC cells are positive for EpCAM (23). A low positive expression rate hampers its use in monitoring patients with tumor metastasis after LT. Negative enrichment, in contrast with positive enrichment, uses antibodies to target unwanted background cells such as leukocytes, which then adhere to magnetic particles, so tumor cells are retained. It has advantages in that it is simple, provides easy-to-use batch separation and allows for high-throughput processing (24). Compared with positive enrichment, it can enrich for all subtypes of CTCs. In addition, the size, density, and charge of CTCs will change due to cell damage and immune attack, and the accuracy of biophysical-related separation methods may be affected. Negative enrichment does not depend on any physical properties of tumor cells, so it can eliminate this effect. ImFISH further improves the sensitivity and signal intensity. In our study, by using this method, CTCs were detected in 26 of 50 (52\%) patients before LT. The sensitivity and specificity of CTCs detection in our study were $83.3 \%$ and $62.5 \%$, respectively. The results showed that this strategy had good sensitivity and specificity so it could be helpful to detect tumors. Schulze et al. revealed that CTCs that were EpCAM (+) had a positive correlation with HCC staging and AFP level (25). In our study, the results showed that CTCs result was correlated with tumor size $\left(\chi^{2}=5.773, \mathrm{P}=0.016\right)$, AFP level $\left(\chi^{2}=5.454, \mathrm{P}=0.020\right)$ and tumor grade $\left(\chi^{2}=6.478, P=0.039\right)$. Our study results supported Schulze's report.

With improvements in CTCs detection methods, most studies have revealed that CTCs are related to HCC recurrence, metastasis, disease-free survival (DFS) and overall survival (OS). Cheng et al. (26) revealed that total CTCs are more effective compared with AFP in the diagnosis of HCC. Our study showed that the preoperative positive rate of CTCs and the postoperative recurrence rate in patients with HCC were $52 \%$ and $24 \%$, respectively. The recurrent rate in patient meeting Milan criteria and UCSF criteria was $8.7 \%$ and $7.1 \%$, respectively. The recurrence time after LT was earlier in CTC-positive patients than in the CTC-negative group $(20.14 \pm 2.13$ and $28.75 \pm 1.56$ months, $\mathrm{P}=0.020$ ). The 1 -year overall survival rate of patients with CTC-positive was smaller than those with CTC-negative, but without significantly difference (88.5\% vs. $91.7 \%, \mathrm{P}=0.751$ ). In multivariate analysis, we found that CTCs result was the only independent impact factor affecting recurrence and preoperative AFP was not significantly different. Sun et al. (27) reported that patients with more CTCs had a worse prognosis after surgery than patients with less CTCs. However, Wang (28) suggested that CTCs levels and subtype were not effective in monitoring HCC recurrence after LT. Our study supported Sun's conclusion. Some reports concluded that LT survival within one year can reach $80 \%$ to $90 \%$ (29). In our study, 1 -year overall survival rate was $88 \%(44 / 50)$, and it was consistent with the current epidemiological results. We didn't observe the effect of CTCs result on survival rate, and the reason would be small sample size and short followup period. Residual CTCs may still cause metastasis or recurrence even after removal of the primary cancer or transplantation, and changes in the number of CTCs during therapy may predict prognosis and recurrence in patients with cancer (30). Therefore, further studies are needed to investigate the predictive value of postoperative CTCs in patients with HCC. 
Our study is limited by its small sample size and by being from a single-center institution. Larger multicenter studies are needed to determine whether CTCs result can predict recurrence in patients with HCC after LT. Second, other tumor types, such as cholangiocarcinoma, were not investigated in this study. In addition, postoperative CTCs were not included in this study. The association between postoperative CTCs and early recurrence of patients with HCC after LT should have been analyzed in the study. For future studies, the 3- and 5-year DFS and OS values should be calculated to obtain more convincing conclusions.

\section{Conclusions}

In conclusion, CTCs result was closely related to the early recurrence of patients with HCC after LT, and patients in the CTC-positive group had a worse prognosis than those in the CTC-negative group. Larger multicenter studies are needed, and longer follow-up outcomes should be used to obtain more convincing results.

\section{Acknowledgments}

Funding: This work was supported by the National Natural Science Foundation of China (81401324 and 81770410), Science and Technology Planning Project of Guangdong Province (2016A020215048), Guangdong Provincial Key Laboratory of Organ Donation and Transplant Immunology (2013A061401007), Guangdong Basic and Applied Basic Research Foundation (2020A1515011557), Guangdong Provincial International Cooperation Base of Science and Technology (Organ Transplantation) (2015B050501002), and Scientific Program for Young Teacher of Sun Yat-sen University (16ykpy05), China.

\section{Footnote}

Reporting Checklist: The authors have completed the STROBE reporting checklist. Available at http://dx.doi. org/10.21037/atm-20-2751

Data Sharing Statement: Available at http://dx.doi. org/10.21037/atm-20-2751

Conflicts of Interest: All authors have completed the ICMJE uniform disclosure form (available at http://dx.doi. org/10.21037/atm-20-2751). The authors have no conflicts of interest to declare.
Ethical Statement: The authors are accountable for all aspects of the work in ensuring that questions related to the accuracy or integrity of any part of the work are appropriately investigated and resolved. The study was conducted in accordance with the Declaration of Helsinki (as revised in 2013) and approved by the Institutional Ethics Committee for Clinical Research and Animal Trials of the First Affiliated Hospital of Sun Yat-sen University and informed consent waiver was granted by the IEC given the retrospective, minimal risk nature of the study \{Approval ID: [2020]088\}.

Open Access Statement: This is an Open Access article distributed in accordance with the Creative Commons Attribution-NonCommercial-NoDerivs 4.0 International License (CC BY-NC-ND 4.0), which permits the noncommercial replication and distribution of the article with the strict proviso that no changes or edits are made and the original work is properly cited (including links to both the formal publication through the relevant DOI and the license). See: https://creativecommons.org/licenses/by-nc-nd/4.0/.

\section{References}

1. Chen $\mathrm{W}$, Zheng R, Baade PD, et al. Cancer statistics in China, 2015. CA Cancer J Clin 2016;66:115-32.

2. Wang JH, Wang CC, Hung CH, et al. Survival comparison between surgical resection and radiofrequency ablation for patients in BCLC very early/early stage hepatocellular carcinoma. J Hepatol 2012;56:412-8.

3. Ferreira MM, Ramani VC, Jeffrey SS. Circulating tumor cell technologies. Mol Oncol 2016;10:374-94.

4. Racila E, Euhus D, Weiss AJ, et al. Detection and characterization of carcinoma cells in the blood. Proc Natl Acad Sci U S A 1998;95:4589-94.

5. Vona $\mathrm{G}$, Sabile A, Louha M, et al. Isolation by size of epithelial tumor cells : a new method for the immunomorphological and molecular characterization of circulatingtumor cells. Am J Pathol 2000;156:57-63.

6. Cristofanilli M, Budd GT, Ellis MJ, et al. Circulating tumor cells, disease progression, and survival in metastatic breast cancer. N Engl J Med 2004;351:781-91.

7. Lara $\mathrm{O}$, Tong $\mathrm{X}$, Zborowski $\mathrm{M}$, et al. Enrichment of rare cancer cells through depletion of normal cells using density and flow-through, immunomagnetic cell separation. Exp Hematol 2004;32:891-904.

8. Harouaka RA, Zhou MD, Yeh Y'T, et al. Flexible micro spring array device for high-throughput enrichment of 
viable circulating tumor cells. Clin Chem 2014;60:323-33.

9. Sangiovanni A, Colombo M. Treatment of hepatocellular carcinoma: beyond international guidelines. Liver Int 2016;36 Suppl 1:124-9.

10. Sapisochin G, Bruix J. Liver transplantation for hepatocellular carcinoma: outcomes and novel surgical approaches. Nat Rev Gastroenterol Hepatol 2017;14:203-17.

11. Quaglia A. Hepatocellular carcinoma: a review of diagnostic challenges for the pathologist. J Hepatocell Carcinoma 2018;5:99-108.

12. Gorgen A, Muaddi H, Zhang W, et al. The New Era of Transplant Oncology: Liver Transplantation for Nonresectable Colorectal Cancer Liver Metastases. Can J Gastroenterol Hepatol 2018;2018:9531925.

13. Dageforde LA, Fowler KJ, Chapman WC. Liver transplantation for hepatocellular carcinoma: current update on treatment and allocation. Curr Opin Organ Transplant 2017;22:128-34.

14. Yao FY, Ferrell L, Bass NM, et al. Liver transplantation for hepatocellular carcinoma: expansion of the tumor size limits does not adversely impact survival. Hepatology 2001;33:1394-403.

15. Mazzaferro V, Regalia E, Doci R, et al. Liver transplantation for the treatment of small hepatocellular carcinomas in patients with cirrhosis. N Engl J Med 1996;334:693-9.

16. Kim WR, Lake JR, Smith JM, et al. OPTN/SRTR 2015 Annual Data Report: Liver. Am J Transplant 2017;17 Suppl 1:174-251.

17. Saidi RF, Hejazi Kenari SK. Liver transplantation for hepatocellular carcinoma: past, present and future. Middle East J Dig Dis 2013;5:181-92.

18. Bhogal RH, Sutaria R, Gunson BK, et al. Similar liver transplantation survival with selected cardiac death donors and brain death donors (Br J Surg 2010; 97: 744-753). Br J Surg 2010;97:1310; author reply 1310-1.

19. Wadei HM, Lee DD, Croome KP, et al. Early Allograft Dysfunction Is Associated With Higher Risk of Renal Nonrecovery After Liver Transplantation. Transplant Direct 2018;4:e352.

Cite this article as: Chen Z, Lin X, Chen C, Chen Y, Zhao Q, Wu L, Wang D, Ma Y, Ju W, Chen M, He X. Analysis of preoperative circulating tumor cells for recurrence in patients with hepatocellular carcinoma after liver transplantation. Ann Transl Med 2020;8(17):1067. doi: 10.21037/atm-20-2751
20. Carr BI. Some new approaches to the management of hepatocellular carcinoma. Semin Oncol 2012;39:369-73.

21. Cristofanilli M, Hayes DF, Budd GT, et al. Circulating tumor cells: a novel prognostic factor for newly diagnosed metastatic breast cancer. J Clin Oncol 2005;23:1420-30.

22. Barbazan J, Dunkel Y, Li H, et al. Prognostic Impact of Modulators of $\mathrm{G}$ proteins in Circulating Tumor Cells from Patients with Metastatic Colorectal Cancer. Sci Rep 2016;6:22112.

23. Yamashita T, Forgues $M$, Wang $W$, et al. EpCAM and alpha-fetoprotein expression defines novel prognostic subtypes of hepatocellular carcinoma. Cancer Res 2008;68:1451-61.

24. Liu Z, Fusi A, Klopocki E, et al. Negative enrichment by immunomagnetic nanobeads for unbiased characterization of circulating tumor cells from peripheral blood of cancer patients. J Transl Med 2011;9:70.

25. Schulze K, Gasch C, Staufer K, et al. Presence of EpCAMpositive circulating tumor cells as biomarker for systemic disease strongly correlates to survival in patients with hepatocellular carcinoma. Int J Cancer 2013;133:2165-71.

26. Cheng Y, Luo L, Zhang J, et al. Diagnostic Value of Different Phenotype Circulating Tumor Cells in Hepatocellular Carcinoma. J Gastrointest Surg 2019;23:2354-61.

27. Sun YF, Xu Y, Yang XR, et al. Circulating stem cell-like epithelial cell adhesion molecule-positive tumor cells indicate poor prognosis of hepatocellular carcinoma after curative resection. Hepatology 2013;57:1458-68.

28. Wang S, Zheng Y, Liu J, et al. Analysis of circulating tumor cells in patients with hepatocellular carcinoma recurrence following liver transplantation. J Investig Med 2018;66:1-6.

29. Meirelles Junior RF, Salvalaggio P, Rezende MB, et al. Liver transplantation: history, outcomes and perspectives. Einstein (Sao Paulo) 2015;13:149-52.

30. Barreto G, Neve R, Shrikhande SV, et al. Role of circulating tumour cells in predicting recurrence after excision of primary colorectal carcinoma (Br J Surg 2007; 94: 96-105). Br J Surg 2007;94:644; author reply 644-5. 